\title{
MAGNETIC PERFORMANCE OF A FAST EXCITATION WIGGLER
}

Juan C. Gallardo, T. Romano, A. van Steenbergen

March 1993 
Magnetic Performance of a Fast Excitation Wiggler

Juan C. Gallardo, T. Romano, A. van Steenbergen

March 1993 


\title{
MAGNETIC PERFORMANCE OF
}

\section{A FAST EXCITATION WIGGLER*}

\author{
Juan C. Gallardo \\ Physics Dep. 901-A, Brookhaven National Laboratory \\ Upton, New York 11973 \\ Tel:516-282-3523; Fax:516-282-3248; e-mail:jcg@bnlcl1.bnl.gov \\ T. Romano, A. van Steenbergen \\ NSLS, 725-B, Brookhaven National Laboratory \\ Upton, New York 11973 \\ Tel:516-282-4604; Fax:516-282-3000; e-mail:avanst@bnlcl1.bnl.gov
}

\begin{abstract}
With the objective of performing an inverse free-electron laser accelerator experiment, an iron dominated (Vanadium Permendur), fast excitation, high $\mathrm{K}$ planar wiggler has been built and measured. We present in this report an analysis of a constant period wiggler and several tapering configurations (gap=4 mm; $3.0 \mathrm{~cm}<\lambda_{w}<5$. $\mathrm{cm}$ ) when we drive it to a peak field of $B_{\max } \approx 1.4 \mathrm{~T}$.
\end{abstract}

\section{INTRODUCTION}

A detailed description of the fast excitation wiggler shown in Figs. 1 and 2 has been given in several papers [1] - [5]; in particular, additional magnetic measurements were presented

*This research was supported by the U.S. Department of Energy under Contract No. DE-ACO276-CH00016. 
in Ref. [6]; Tb. I presents : summary of the different wiggler configurations that have been studied up to now. In this note we discuss the field errors and the imposed constraints to maintain electron and laser overlap.

The experimental setup allows the measurement of the vertical component of the wiggler magnetic field in the horizontal plane at different vertical and horizontal positions in the gap, $B_{y}\left(x, y, z_{j}\right)$ whəre $z_{j}=\Delta j$ with $\Delta=0.15875 \mathrm{~cm}$ corresponding to a complete turn of the driven shaft of the traveling measurement system. The relative error in position of the probe is estimated to be around $0.02 \mathrm{~cm}$ and the estimated error in the induced voltage signal after being integrated is about $0.1 \%$.

To calculate the electron trajectory in this real wiggler it is necessary to make a complete reconstruction of the field in the gap from the measured values. From general principles [7] we express the magnetic scalar potential as

$$
\Phi(x, y, z)=X(x) Y(y) Z(z)
$$

with $\vec{B}(x, y, z)=-\vec{\nabla} \Phi(x, y, z)$.

If we assume symmetry in the horizontal plane and periodicity in the longitudinal direction then $X(x)=X(-x)$ and $Z(z)=\cos n k z$ where $k=\frac{2 \pi}{\lambda}$ with $\lambda$ the period, we can write the most general expression for the scalar potential as

$$
\Phi(x, y, z)=\sum_{n=1}^{\infty} \cosh \left(n k_{1} x\right)\left[A_{n} \exp \left(n k_{2} y\right)+B_{n} \exp \left(-n k_{2} y\right)\right] \cos \left(n k_{3} z\right)
$$

with the constraint $k_{3}^{2}=k_{1}^{2}+k_{2}^{2} \equiv k_{2}^{2}\left(1+r^{2}\right)$.

Notice that there is no median plane symmetry and the deviation can be represented with a single parameter $\eta$ such that,

$$
B_{y}(x,-g, z)=\eta B_{y}(x, g, z)
$$

where $\mathrm{g}$ is the gap distance. This relation can be understood making use of the Gauss theorem in the transverse plane (see Fig. 1).

Simple algebraic manipulations lead to the expression of the field in the gap, 


$$
\begin{aligned}
& B_{x}(x, y, z)=n k_{1} \sinh \left(n k_{1} x\right)\left[A_{n} \exp \left(n k_{2} y\right)+B_{n} \exp \left(-n k_{2} y\right)\right] \cos \left(n k_{3} z\right) \\
& B_{y}(x, y, z)=n k_{2} \cosh \left(n k_{1} x\right)\left[A_{n} \exp \left(n k_{2} y\right)-B_{n} \exp \left(-n k_{2} y\right)\right] \cos \left(n k_{3} z\right) \\
& B_{z}(x, y, z)=-n k_{3} \cosh \left(n k_{1} x\right)\left[A_{n} \exp \left(n k_{2} y\right)+B_{n} \exp \left(-n k_{2} y\right)\right] \sin \left(n k_{3} z\right)
\end{aligned}
$$

the coefficients $A_{n}$ and $B_{n}$ are determined by the boundary conditions at the poles and the peak vertical magnetic field on axis $B_{\text {on }}$; they read,

$$
\begin{aligned}
& A_{n}=\frac{B_{o n}}{2 n k_{2}(1+\eta) \sinh \left(n k_{2} d\right)}\left[\exp \left(n k_{2} d\right)-\eta \exp \left(-n k_{2} d\right)\right] \\
& B_{n}=\frac{B_{o n}}{2 n k_{2}(1+\eta) \sinh \left(n k_{2} d\right)}\left[\exp \left(-n k_{2} d\right)-\eta \exp \left(n k_{2} d\right)\right]
\end{aligned}
$$

Next we use the fields so determined to calculate the electron trajectory in the wiggler field, the spontaneous emission spectrum and by means of the Madey's theorem [8], the free-electron laser small-signal gain.

\section{PERFORMANCE OF A CONSTANT PERIOD FAST EXCITATION WIGGLER}

In Fig. 3 we show $B_{o}(z)$ vs. $z$ measured on axis for a current of $6 k A$. In Figs. $4 a$ and $4 \mathrm{~b}$ we display the scattering of positive and negative peak fields. Notice that there is an apparent negative dipole offset. It has been verified that this is $/$ ue to a small $(0.35 \%)$ systematic error related to the unequal response of the field measurement integrating circuit to poritive and negative magnetic field.

The rms-fluctuation of the peak field $\sigma_{B}=\sqrt{\left\langle\frac{\delta B^{2}}{B^{2}}\right\rangle}$ about the average is under $0.15 \%$ and the maximum scatter is $1.0 \%$.

A complete measured field representation $B_{y}(x, y, z)$ for a single pole region $(g=$ $4 \mathrm{~mm}, 0.5 \lambda=18.8 \mathrm{~mm}, I=6 \mathrm{kA}$ ) is given in Fig. 5 for $-1.2 \leq y \leq 1.2 \mathrm{mmm}$ and $-6.0 \leq x \leq 6.0 \mathrm{~mm}$.

A Fourier transform analysis of the entire field map on axis has been performed; in Fig. 6 we show the power spectrum in log scale computed using the Fast Fourier Transform routine SSWD from the IMSL library with a Parzen window. From the power spectrum plot, the odd harmonics up to order 7 th are clearly in evidence, a natural consequence of the periodicity 
of the field. The magnitude of the noise level is about $10^{-6}$ and up to that level there is no indication of even harmonics. The 3rd. harmonic is about $3 \%$ of the fundamental and the magnitude of the odd harmonics is fitted by a Laurent series $-0.01+\frac{0.26}{n}-\frac{2.1}{n^{2}}+\frac{6.1}{n^{3}}-\frac{3.3}{n^{4}}$ in reasonable agreement with expectations.

The normalized horizontal velocity $x^{\prime}=\frac{v_{x}}{v_{z}}$ is given essentially by the first integral of the vertical component of the magnetic field $B_{y}(0,0, z)=B_{o}(z)$, i.e.,

$$
x^{\prime} \approx \frac{K}{\gamma} \frac{2 \pi}{\lambda_{w}} \int_{-\infty}^{z} d z^{\prime} \frac{B_{o}\left(z^{\prime}\right)}{B^{\max }}
$$

likewise, the position $\mathrm{x}$ is given by the second integral of the field,

$$
x \approx \frac{K}{\gamma} \frac{2 \pi}{\lambda_{w}} \int_{-\infty}^{z} d z^{\prime}\left(z-z^{\prime}\right) \frac{B_{o}\left(z^{\prime}\right)}{B^{\max }}
$$

where we have introduced $K=\frac{e B^{\max } \lambda_{w}}{m c^{2} \beta_{z} 2 \pi}=0.934 B^{\max }[T] \lambda_{w}[\mathrm{~cm}]$, where $B^{\max } \approx 1.4 T$ is the peak field and $\lambda_{w} \approx 3.754 \mathrm{~cm}$.

Figs. 7 and 8 depict $x^{\prime}$ vs. $z$ and $x$ vs. z. We observe that the maximum $x$ oscillation amplitude is $\approx 0.4 \mathrm{~mm}$. The displacement and steering of the beam at the exit of the wiggler are approximately $0.001 \mathrm{~mm}$ and $3.4 \mathrm{mrad}$, respectively.

Magnetic field statistical errors due to imperfections in the fabrication and assembly of the wiggler perturb the electron bsam and leads to a deviation from the trajectory in an ideal (error free) wiggler. This deviation decreases the free-electron laser gain as a result of: a) loss of overlap between laser and electron beams, as a consequence of the electron random walk; b) loss of the resonance condition, as a consequence of the random phase variations. Several authors [9], [10] have shown that the centroid motion of the electron beam satisfy the relation (for uncompensated wigglers)

$$
<\delta x^{2}>=\frac{2}{3} \frac{K^{2} k_{w}^{2}}{2 \gamma}<\left(\frac{\delta B_{y}}{B_{y}}\right)^{2}>\frac{\lambda_{w}}{2}\left(N \lambda_{w}\right)^{3}
$$

and for a compensated wiggler $\left\langle\delta x^{2}\right\rangle$ is reduced by a factor of 4 . The condition to minimize the random walk effect on the gain is to limit $\sqrt{\left.<\delta x^{2}\right\rangle} \leq 0.1 r_{o}$ where $r_{o}$ is the laser beam waist size. For our case $\mathrm{E}_{1} .8$ gives $\sqrt{\left\langle\left(\frac{\delta B_{y}}{B_{y}}\right)^{2}\right\rangle} \approx 0.24 \%$ which is larger than the measured dispersion of $0.15 \%$. 
Similarly, the random phase deviation is given by,

$$
<\delta \psi>=-\frac{K^{2} k_{w}^{3}}{\left(1+\frac{1}{2} K^{2}\right)}<\left(\frac{\delta B_{y}}{B_{y}}\right)^{2}>\frac{\lambda_{w}\left(N \lambda_{w}\right)^{2}}{8} .
$$

Degradation of the FEL gain becomes noticeable when $\langle\delta \psi>\approx \pi$. For our set of param-

eters, the limiting value of $\sqrt{\left\langle\left(\frac{\delta B_{y}}{B_{y}}\right)^{2}\right\rangle} \approx 0.64 \%$ is obtained, which is well met by the wiggler results cited above.

For completness we also present in Fig. 9, a plot of the field $B_{o}(z)$ vs. $z$ for a tapered period wiggler with $2.98 \mathrm{~cm}<\lambda_{w}<5.04 \mathrm{~cm}$ and a current of $6 \mathrm{kA}$.

\section{SPONTANEOUS EMISSION}

The spontaneous emission spectrum of an undulator exhibit sharp peaks at $\omega_{n}=$ $n \omega_{1}(\theta), \quad n=1,2,3, \ldots$ where

$$
\omega_{1}(\theta)=\frac{2 \gamma^{2}}{\left(1+\frac{1}{2} K^{2}+\gamma^{2} \theta^{2}\right)} \omega_{w}
$$

The undulator frequency is $\omega_{w}=\frac{2 \pi c}{\lambda_{w}}$ and $\theta$ is the observation angle with respect to the oscillation plane $(x-z)$. We stress that only in the forward direction, $\theta=0$, we expect to have odd harmonics, at any other angle the spectrum will also show even peaks.

Likewise, the spontaneous emission spectrum of a wiggler exhibit sharp peaks at the harmonics $\omega_{n}(\theta)$ and the spectral flux (number of photons per unit time and per unit solid angle) into a small bandwidth $\Delta \omega$ is given by,

$$
\frac{1}{\hbar \omega} \frac{d^{2} W}{d \Omega^{2}} \equiv \frac{d^{2} \mathcal{F}}{d \Omega^{2}}=\alpha N^{2} \gamma^{2}\left(\frac{\Delta \omega}{\omega}\right) \frac{I}{e} F_{n}(K)
$$

where $\alpha=\frac{1}{137}$ is the fine-structure constant and the function $F_{n}$ is defined as

$$
F_{n}(K)=\frac{K^{2} n^{2}}{\left(1+\frac{1}{2} K^{2}+\gamma^{2} \theta^{2}\right)^{2}} \times\left\{\begin{array}{c}
\left(J_{\frac{1}{2}(n-1)}(n \zeta)-J_{\frac{1}{2}(n+1)}(n \zeta)\right)^{2} \quad n=1,3,5 \ldots \\
4\left(\frac{\gamma \theta}{K}\right)^{2} J_{\frac{1}{2} n}(n \zeta)^{2} \quad n=2,4,6 \ldots
\end{array}\right.
$$

In the cquation above we have used $\zeta=\frac{K^{2}}{4\left(1+\frac{1}{2} K^{2}+\gamma^{2} \theta^{2}\right)^{2}}$. In practical units the spectral flux is 


$$
\frac{d^{2} \mathcal{F}}{d \Omega^{2}}=1.74 \times 10^{14} N^{2} E_{e}[G e V] I[A] F_{n}\left(K^{\prime}\right)
$$

We plot in Fig. 10 the function $F_{n}(K)$ for odd harmonics; it shows that for high values of $\mathrm{K}$ the higher harmonics become more prominent and that the maximum [11] is achicved at the critical frequency $\omega_{c}=\frac{3}{2} \frac{\gamma^{2} 2 \pi c K}{\lambda_{\omega}}$ and the corresponding harmonic number is $n_{c}=\frac{\omega_{c}}{\omega_{1}}=$ $\frac{3}{4} K\left(1+\frac{1}{2} K^{2}\right) \approx 50 ;$ as a consequence, we expect to see in the spontaneous emission spectrum sharp peaks at harmonics of $\omega_{1}(0)$ with a rather broad maximum at harmonics $n_{c} \approx 50$.

In Figs. 11 we plot the spectral flux in arbitrary units near the fundamental $\omega_{1}$ as computed by the code SIN-LUCE [12]. Notice the second harmonics with an intensity $50 \%$ below the fundamental, signaling a misalignment of the electron beam with the magnetic axis of the wiggler. This misalignment is caused by a combination of wiggler errors and improperly matched electron beam. Lastly, in Fig. 12 we show the gain curve at the fundamental frequency $\omega_{1}(0)$.

\section{CONCLUSION}

It has been shown that the fast excitation driven, laminated Vanadium Permendur, wiggler with periodic interleaving of conductive copper field reflectors is capable of satisfying both the FEL and IFEL requirements. As indicated, a specific tapered period slope can readily be achieved. The wiggler structure, as presently executed is robust and simple to assemble and lends itself for easy modifications of period length, field magnitude and taper. These are very important features in actual FEL and IFEL experiments.

\section{ACKNOWLEDGMENTS}

The authors wish to acknowledge the contribution of J. Armendariz to pirt of the experimental work. We are also grateful to L. Giannessi for making the code SIN-LUCE available to us and for his continuous advice and assistance. This research was supported by the U.S. Department of Energy under Contract No. DE-ACO2-76-CH00016. 


\section{REFERENCES}

[1] A. van Steenbergen, patent No.368618, (submitted June 1989) issued in August 1990.

[2] A. van Steenbergen, J. Gallardo, T. Romano, M. Woodle, Fast Excitation variable period wiggler, Proc. PAC San Francisco, IEEE Nucl. Sci., pag. 2724, May 1992.

[3] A. van Steenbergen, J. Gallardo, T. Romano, M. Woodle, Fast excitation wiggler development, Proc. Workshop Prospects for a 1 AFEL, Sag Harbor, N.Y., pag. 79, April 1990; BNL Report BNL-52273.

[4] E. Courant, A. Fisher, J. Gallardo, C. Pellegrini, J. Rogers, J. Sandweiss, J. Sheehan, A. van Steenbergen, S. Ulc, M. Woodle, Inverse Free-Electron Laser Accelerator Development, The Accelerator Test Facility Users' Meeting, October 15-16 1991, BNL Report BNL-47000, CAP \#81, ATF-91P.

[5] A. Fisher, J. Gallardo, J. Sandweiss, A. van Steenbergen, Inverse free-electron laser accelerator, Proc. Port Jeff. Workshop June 1992; to be published.

[6] J. Armendariz, J. Gallardo, T. Romano, A. van Steenbergen, Fast excitation wiggler field measurement results, BNL Report BNL-47928, 1992.

[7] G. Dattoli and A. Renieri, Experimental and Theoretical aspects of the free-electron laser, Laser Handbook, vol. 4, North-Holland, Amsterdam (1985).

[8] J. M. J. Madey, Nuovo Cimento, vol 50B, 64 (1979).

[9] B. Kincaid, J. Opt. Soc. Am., vol B2, 1294 (1985).

[10] J. Gallardo, J. Appl. Phys., vol 170, 1115 (1991) and references therein.

[11] Kwang-Je Kim, Characteristics of Synchrotron Radiation, A.P.S. Conference Proceedings, vol 184, 567 (1989).

[12] R. Barbini, F. Ciocci, G. Dattoli and L. Giannessi, Fortran 77 SIN-LUCE code, private communication (1992). 


\section{TABLES}

TABLE I. Table of wiggler configurations studied. Data are normalized to $L_{w}=24 \mathrm{~cm}$ and $\underline{\underline{I_{e x c}}=4 \mathrm{kA}}$

\begin{tabular}{cccccccc}
\hline \hline$\frac{d B}{d z}[\mathrm{kG} / \mathrm{cm}]$ & $\lambda_{w}[\mathrm{~cm}]$ & $\mathrm{W}_{p}[\mathrm{~cm}]$ & $(\mathrm{r})^{\mathrm{a}}$ & $\left(\mathrm{B}_{i}\right)^{\mathrm{b}}[\mathrm{kG}]$ & $\left(\mathrm{B}_{e}\right)^{\mathrm{c}}[\mathrm{kG}]$ & $\frac{d B}{d z}[\mathrm{kG} / \mathrm{cm}]$ & $\frac{d r}{d z}[1 / \mathrm{cm}]$ \\
\hline WIi & 2.98 & 1.19 & 0.798 & 9.57 & - & $0.0168^{\mathrm{d}}$ & $\approx 0$ \\
var. $\lambda_{w} ;$ const $\mathrm{r}$ & 4.29 & 1.73 & 0.806 & - & 9.97 & - & - \\
\hline WIe & 4.29 & 1.73 & 0.806 & 9.97 & - & $0.0168^{\mathrm{d}}$ & $\approx 0$ \\
var. $\lambda_{w} ;$ const $\mathrm{r}$ & 5.04 & 1.98 & 0.786 & - & 10.37 & - & - \\
\hline WII & 3.96 & 1.07 & 0.540 & 10.05 & - & $0.0168^{\mathrm{d}}$ & 0.006 \\
const $\lambda_{w}$ & 3.96 & 1.37 & 0.692 & - & 10.45 & - & - \\
\hline WIII & 3.25 & 1.02 & 0.628 & 10.11 & - & 0.0072 & -0.006 \\
const $\mathrm{W}_{p}$ & 4.27 & 1.02 & 0.478 & - & 10.28 & - & - \\
\hline WIVi & 3.76 & 1.52 & 0.808 & 10.06 & - & $\pm 0.0068^{\mathrm{e}}$ & $\approx 0$ \\
const $\lambda_{w}, \mathrm{r}$ & 3.76 & 1.52 & 0.808 & - & 9.89 & - & - \\
\hline \hline
\end{tabular}

${ }^{a} r=\frac{W_{p}}{\left(\lambda_{w} / 2\right)}, W_{p}$ denotes the thickness of the VaP.

${ }^{\mathrm{b}} B_{i}=B(z=0)$ except for WIV where $B_{i}=B(z=24)$

${ }^{\mathrm{c}} B_{e}=B(z=24)$ except for WIV where $B_{e}=B(z=0)$

d without partial saturation, 0.0145

${ }^{e}$ end effects 

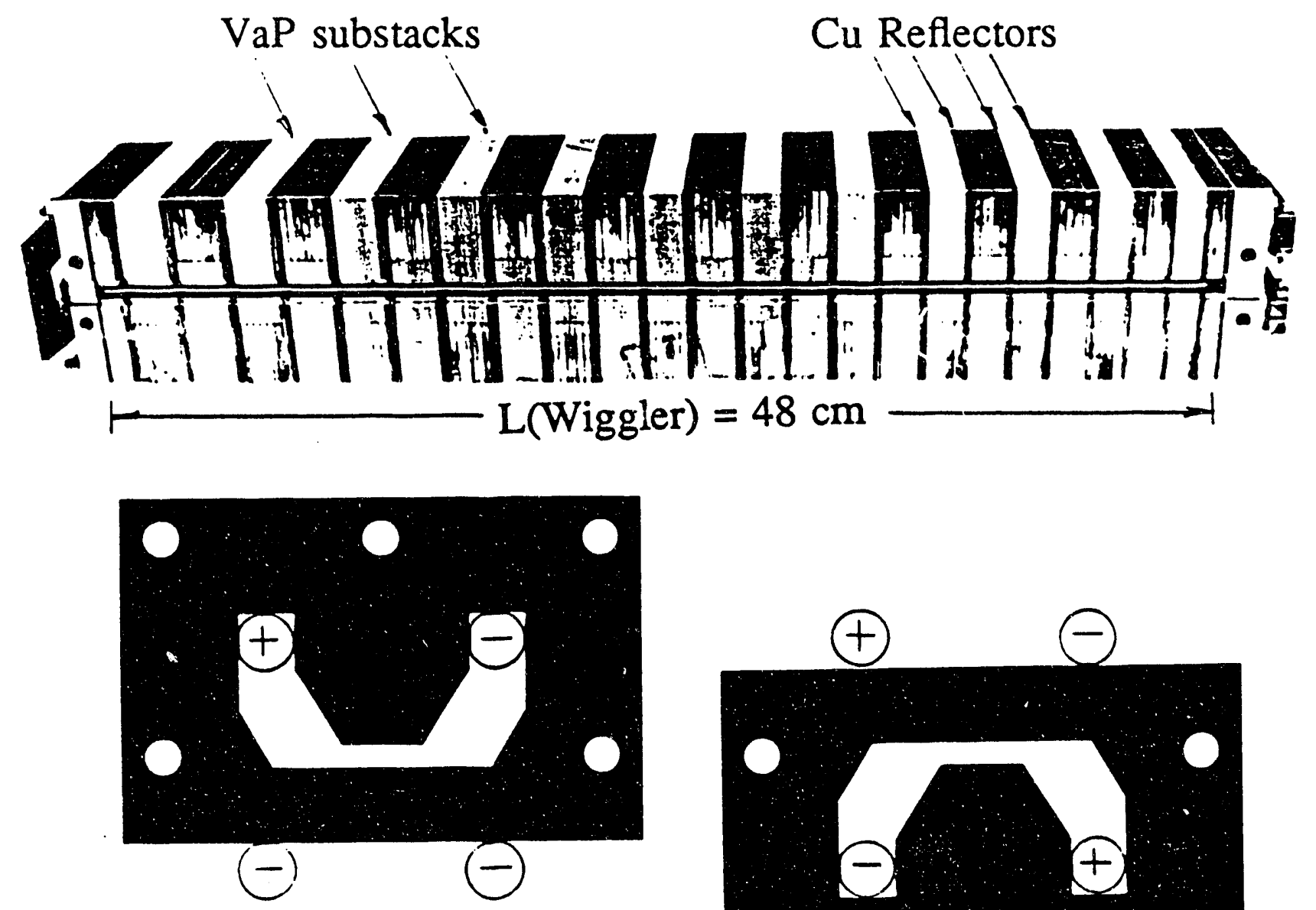

VaP laminations th. $=0.254 \mathrm{~mm}$

$\oplus \quad \Theta$

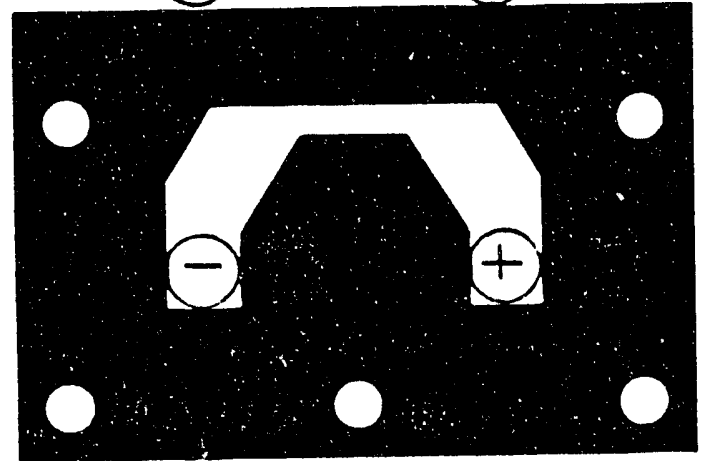

Fast Excitation, Variable Period, Wiggler (IFEL Accelerator, Wiggler Prototype) Stackable, Interleaved VaP laminations and $\mathrm{Cu}$ eddy current "field reflectors"

Fig.1 
Fig.2, "Exploded View" Fast Excitation Variable Period Wiggler [Stackable Laminated Structure]
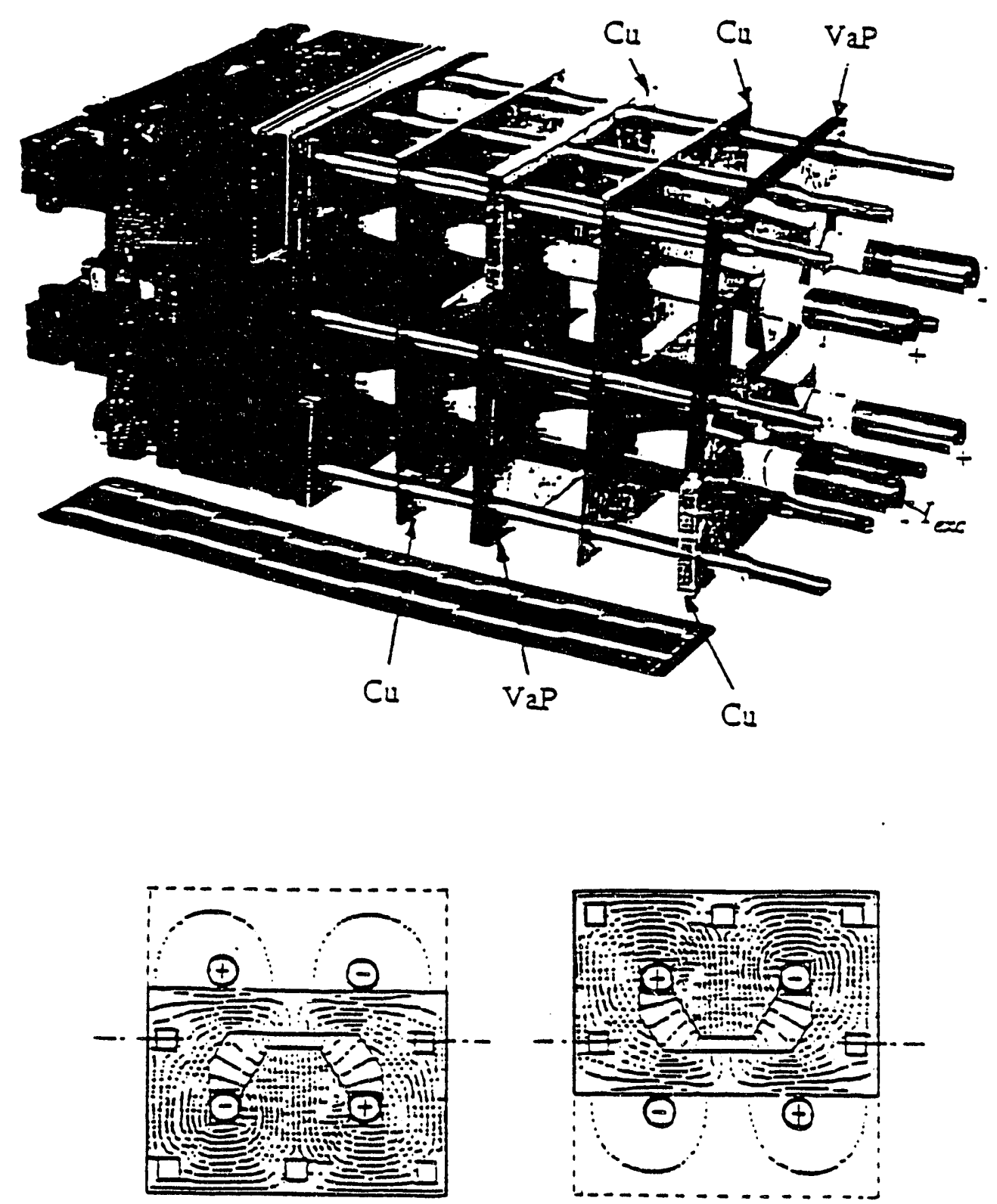

Computed 2D Field Distribution (Excit. current 10kA) 
Excitarion current $6 \mathrm{kA}$

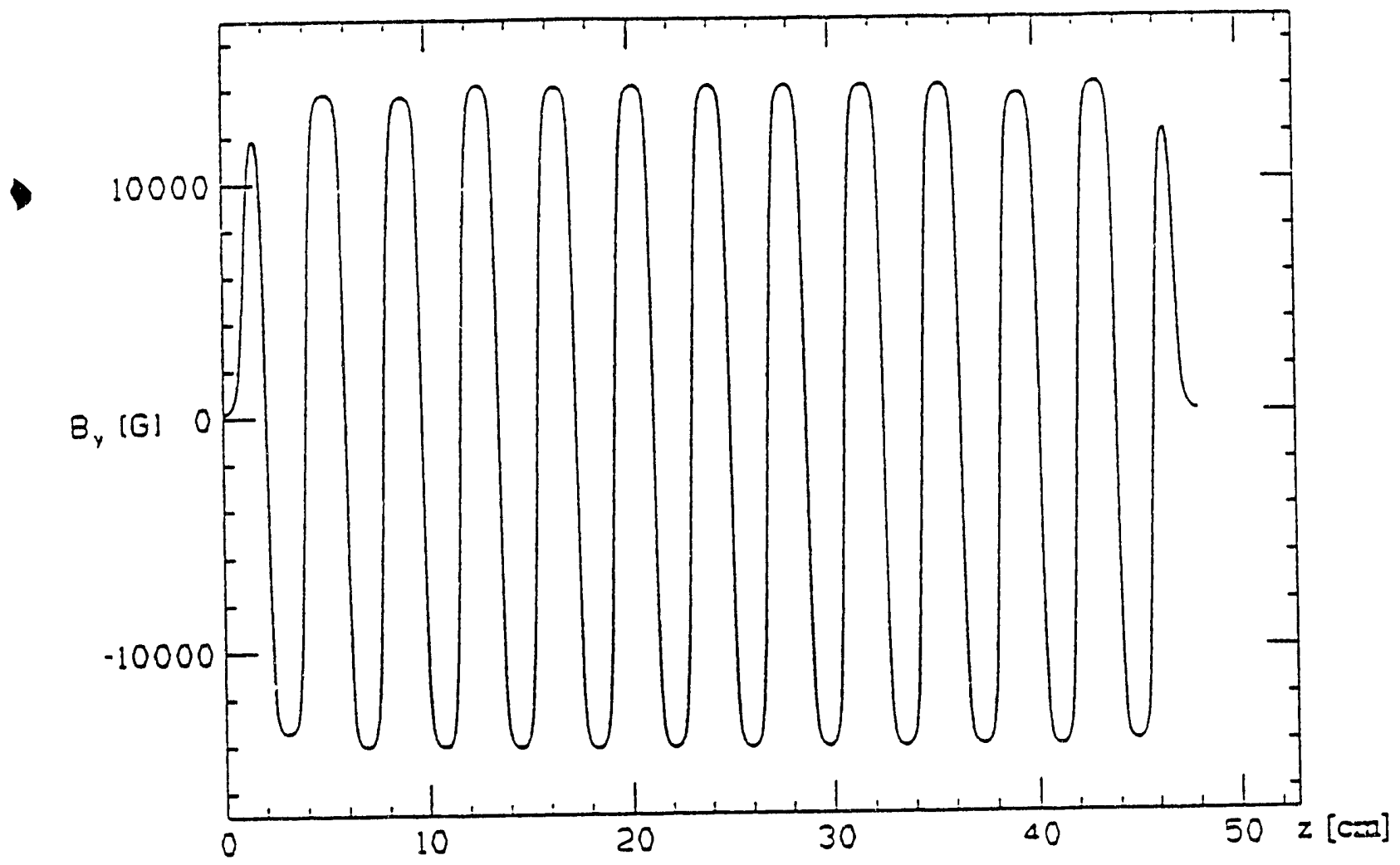

Fig.3 , Constant Period, Fast Exciration, Wiggler

$$
\lambda=3.76 \mathrm{~cm}, \mathrm{~g}=0.40 \mathrm{~cm}
$$

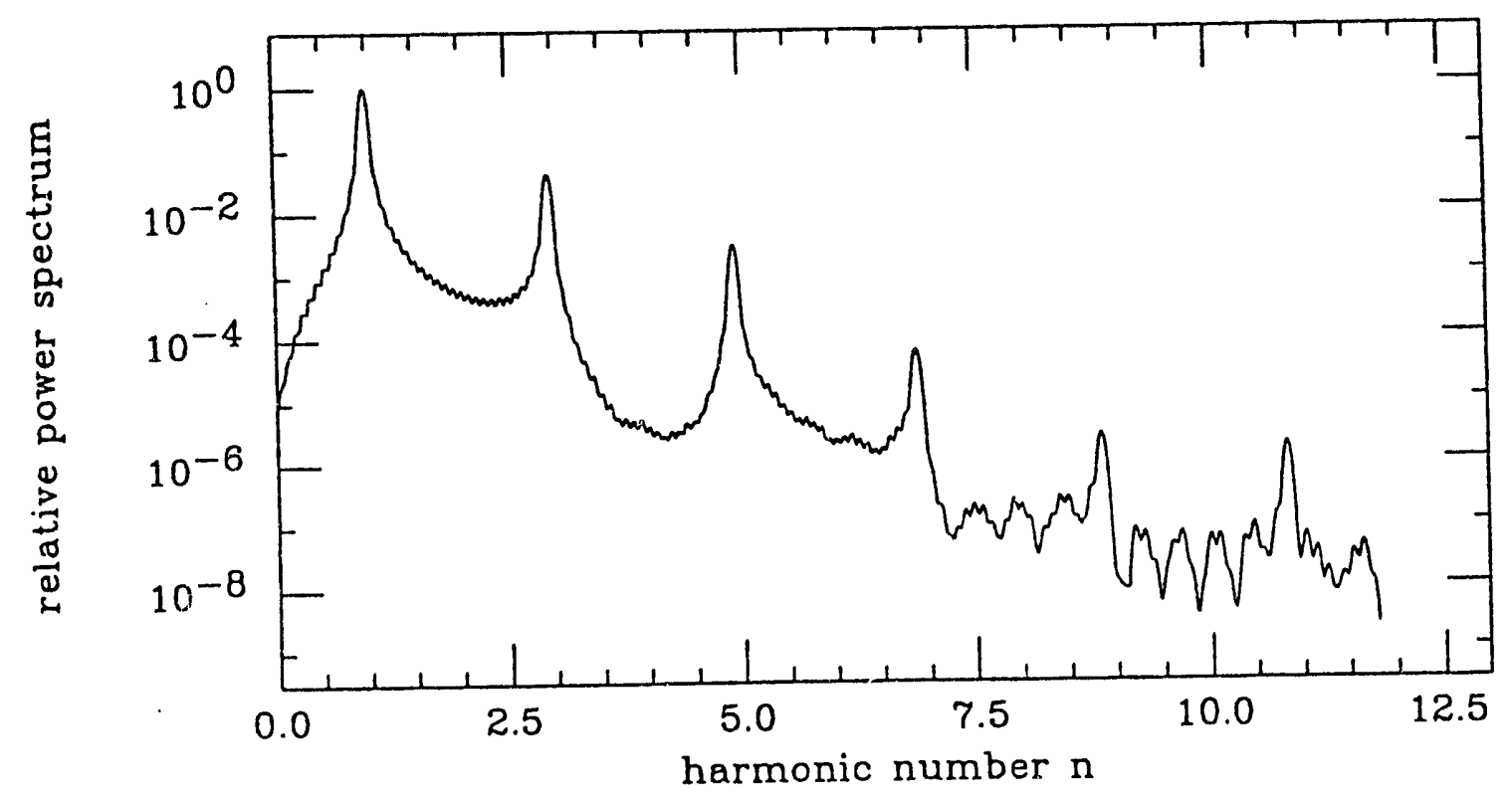

Fig.6, Power Spectum. Constant Period Fast Excitation Wiggle:

$$
\lambda=3.76 \mathrm{~cm}, g=0.40 \mathrm{~cm}, I(\text { exc })=6 \mathrm{kA}
$$


Fig.4a, Positive $B(\max )$ values $(I=6 \mathrm{kA})$
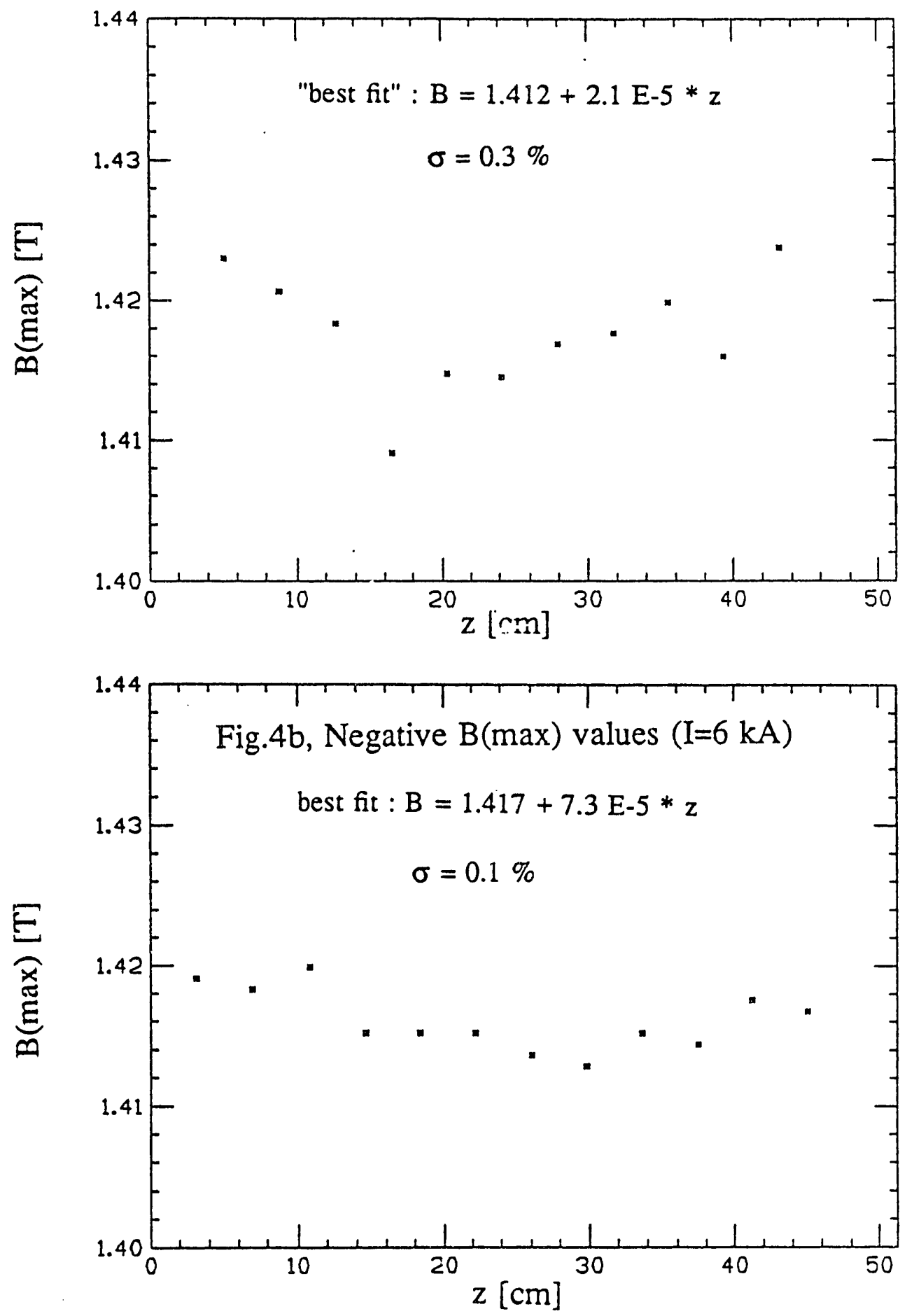

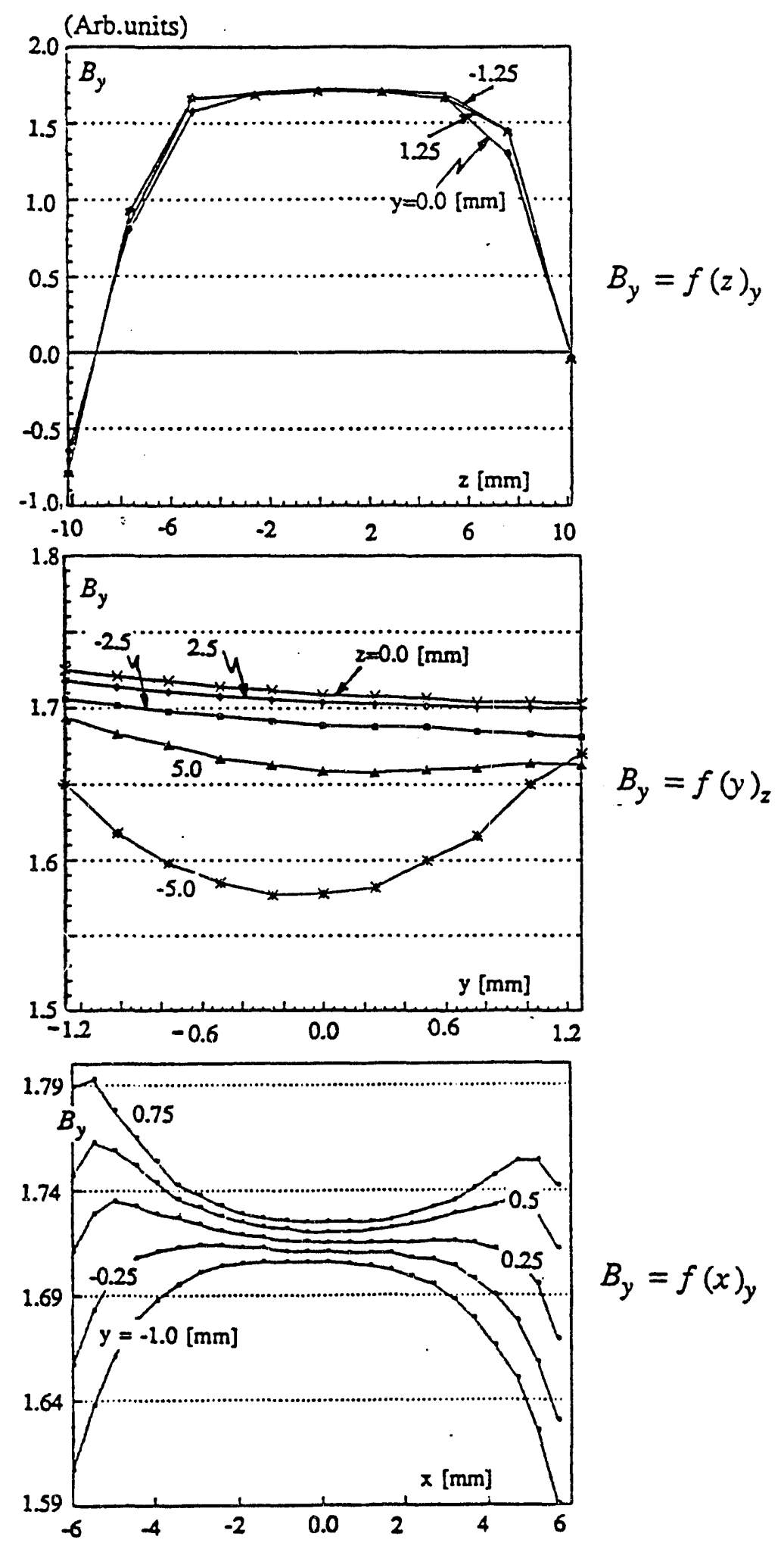

Wiggler Field Distribution $\left(\lambda_{w}=3.76 \mathrm{~cm}, \mathrm{~g}=4 \mathrm{~mm}, I_{\text {exc }}=6 \mathrm{kA}\right)$

Fig.5 
Fig.7

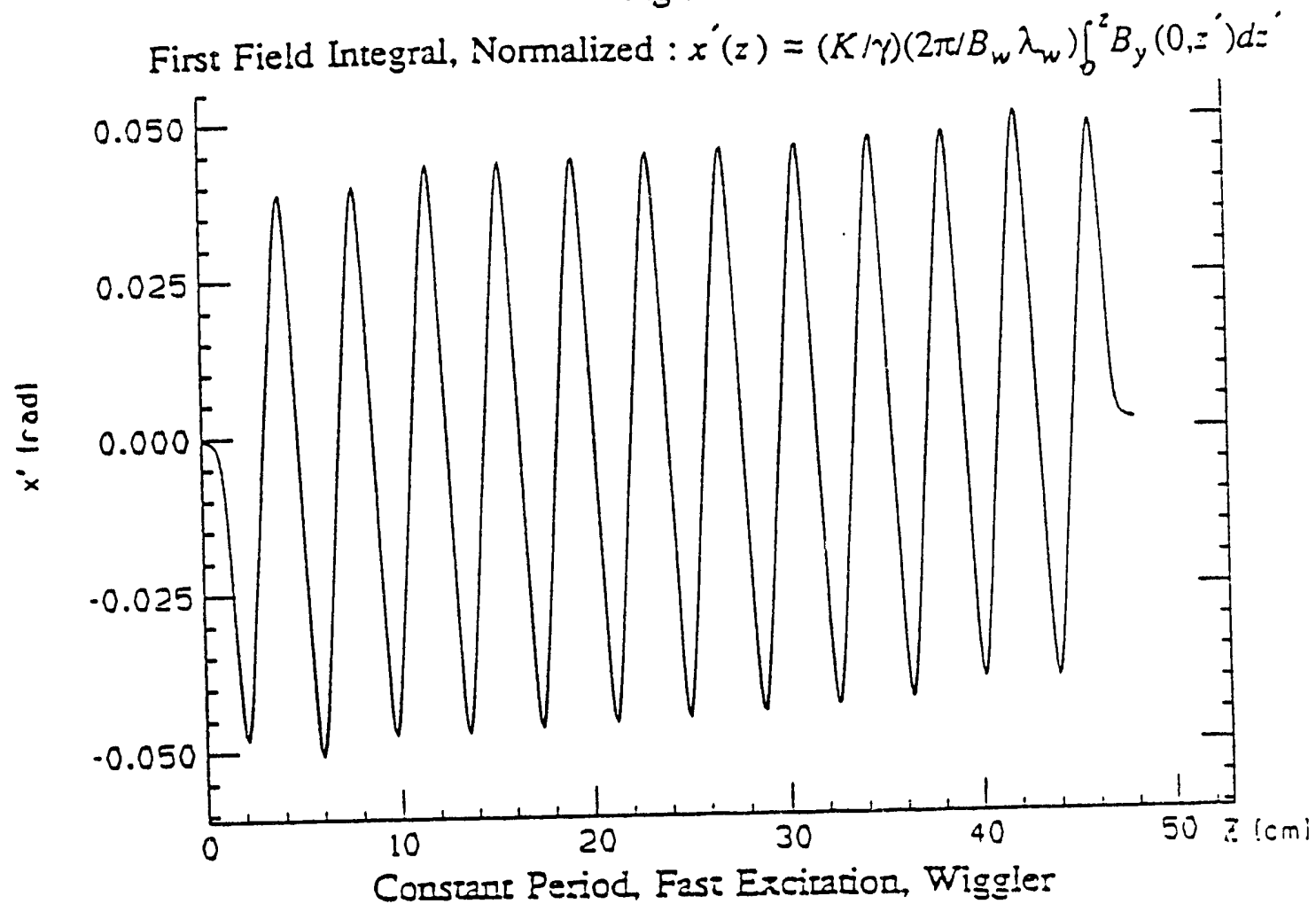

$$
\lambda=3.76 \mathrm{~cm}, \mathrm{~g}=0.40 \mathrm{~cm}
$$

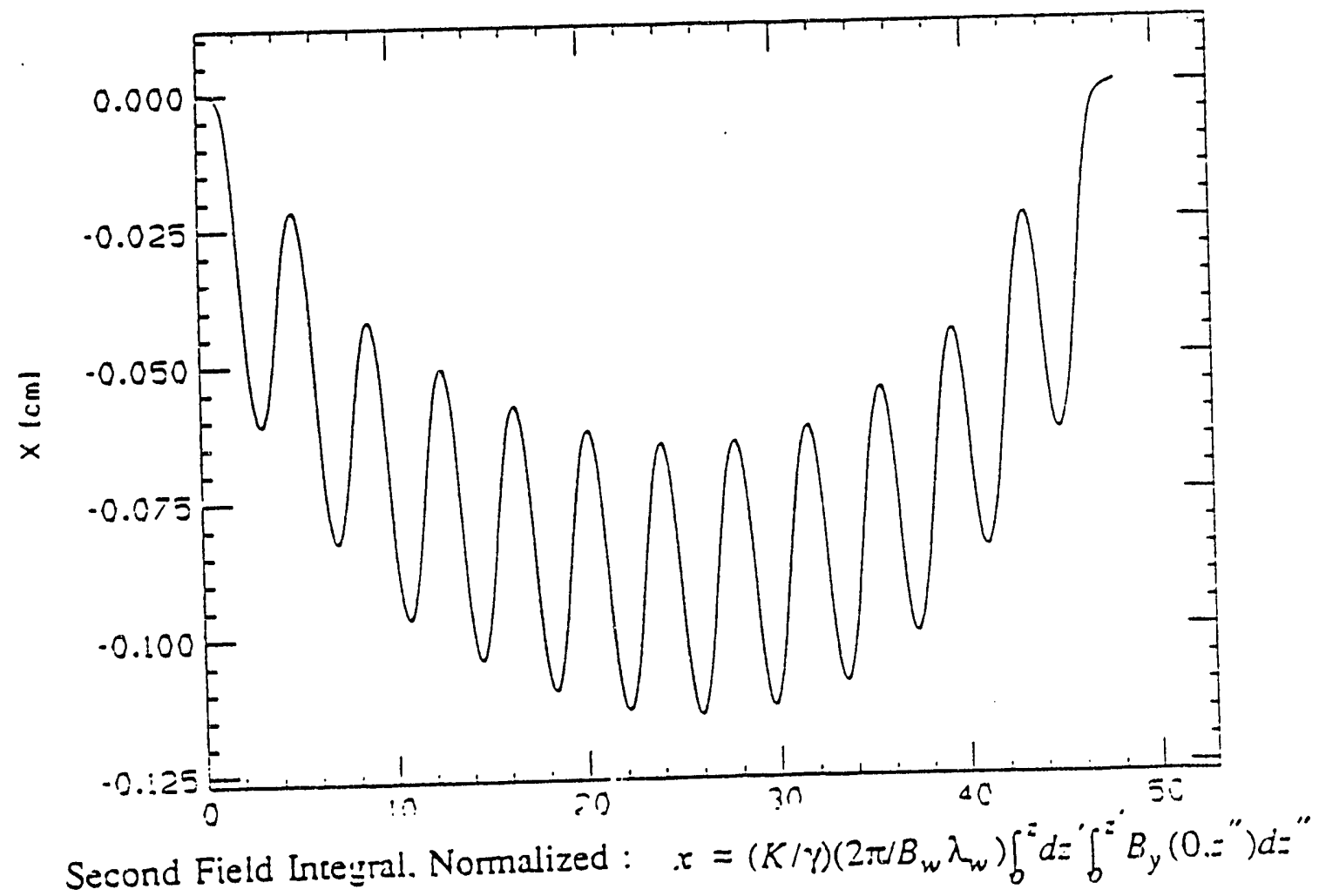

Fig. 8 


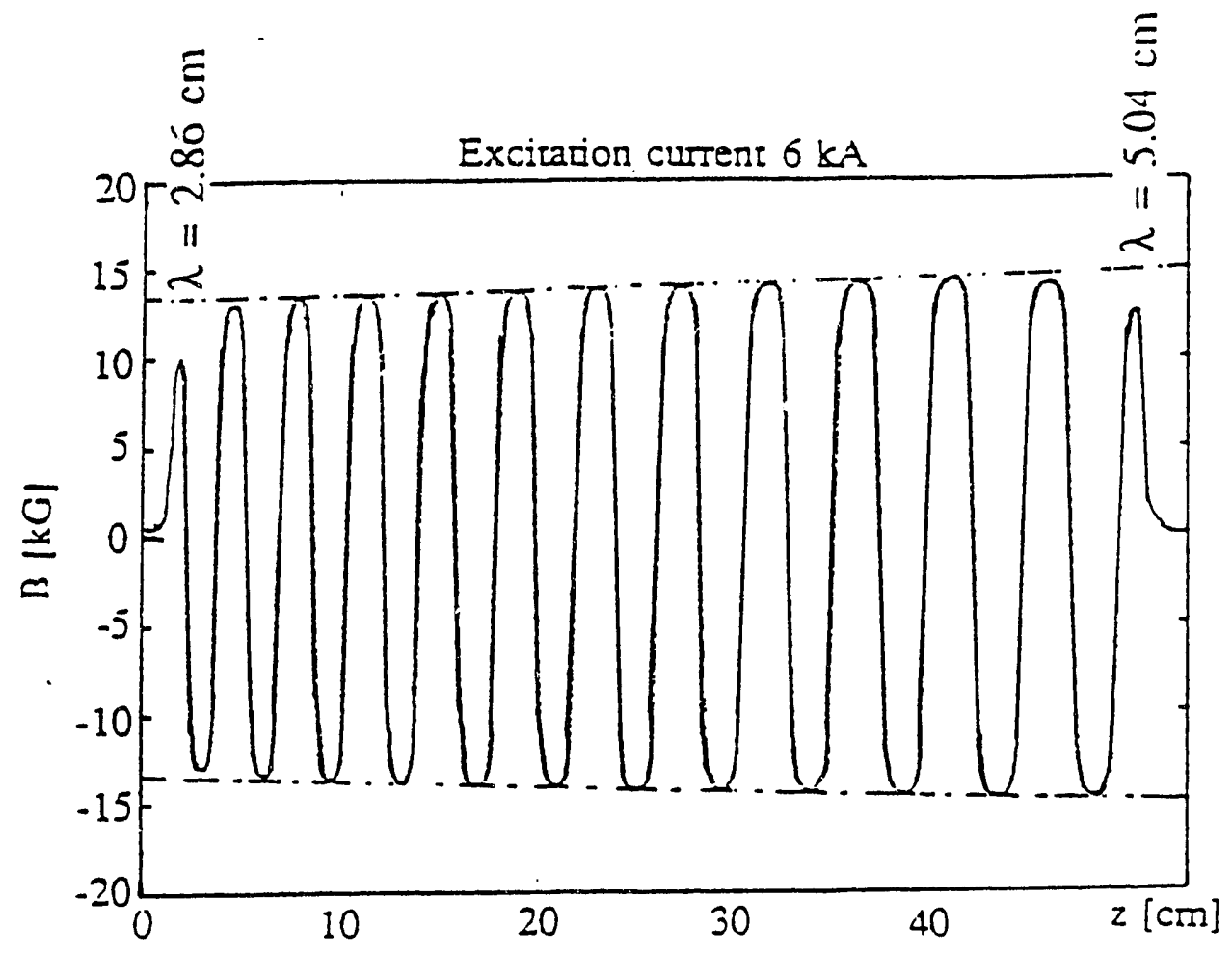

Fig.9.

Variable Period, Fast Excitation, Wiggler (IFEL Accelerator, Wiggler Prototype)

$\lambda=2.98-5.04 \mathrm{~cm}, d \lambda d z=4 \%, g=0.40 \mathrm{~cm}$ 


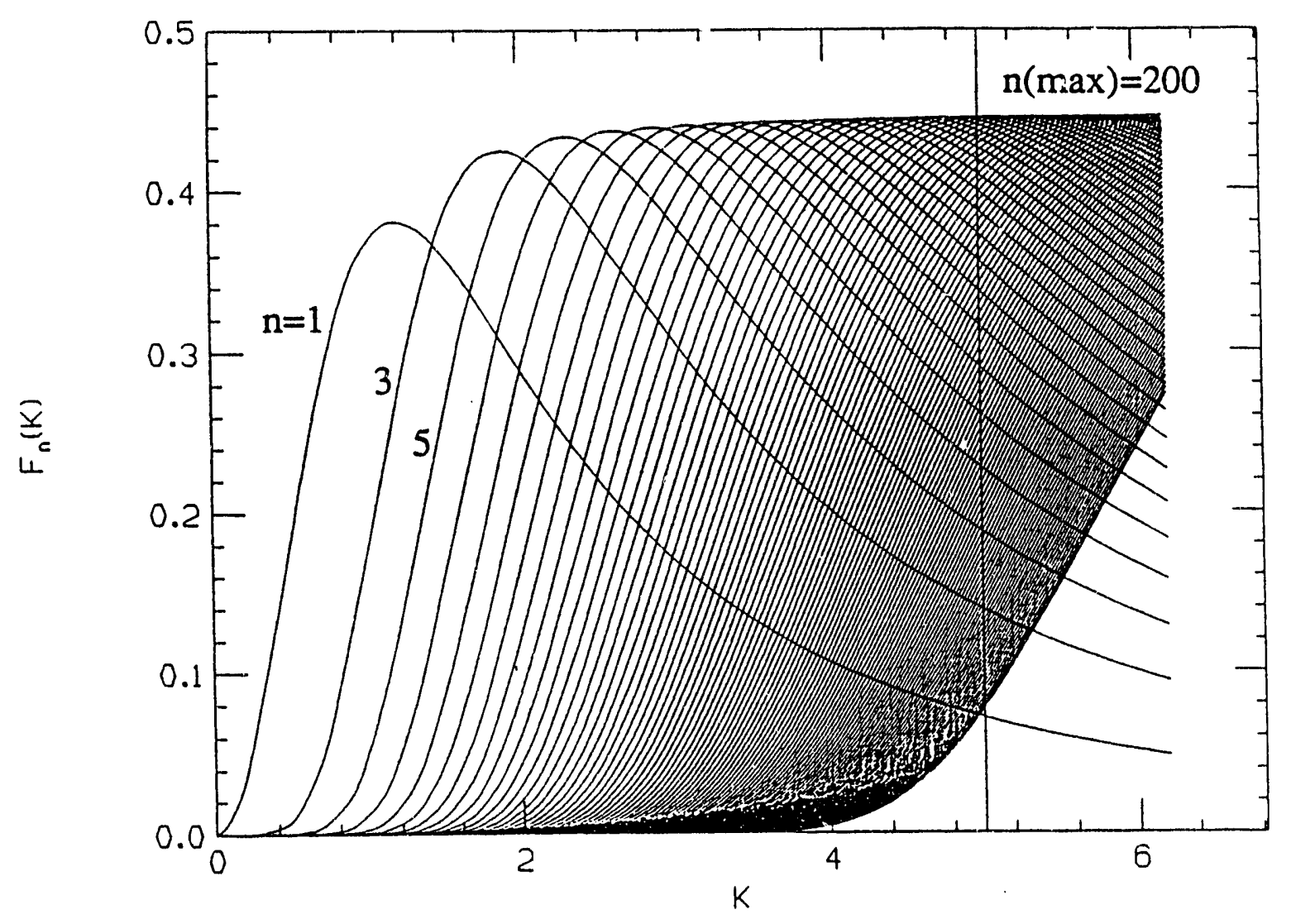

Fig.10, Function $F_{n}(K)$ 


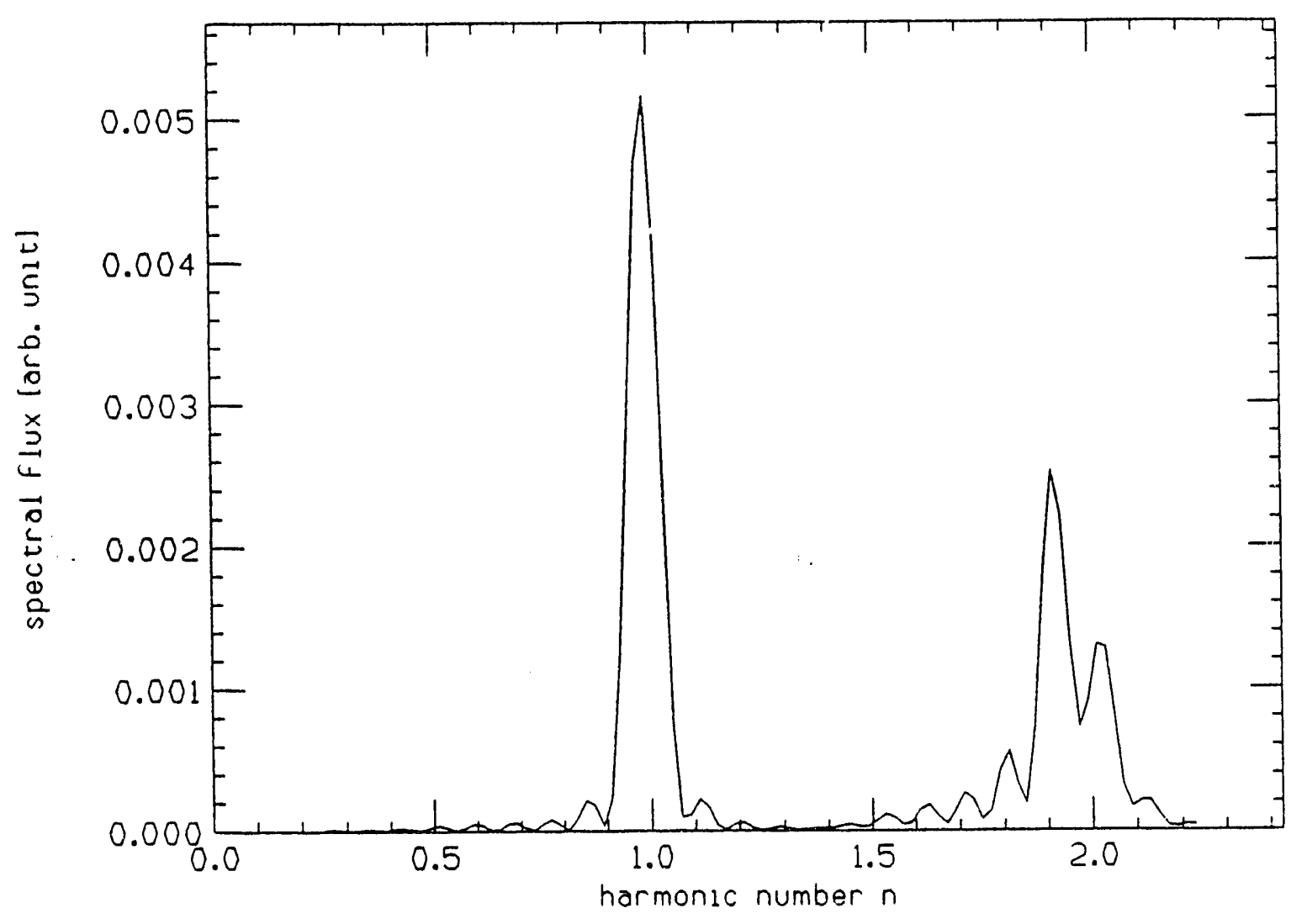

Fig.11, Spectral Flux near $n=1$ 


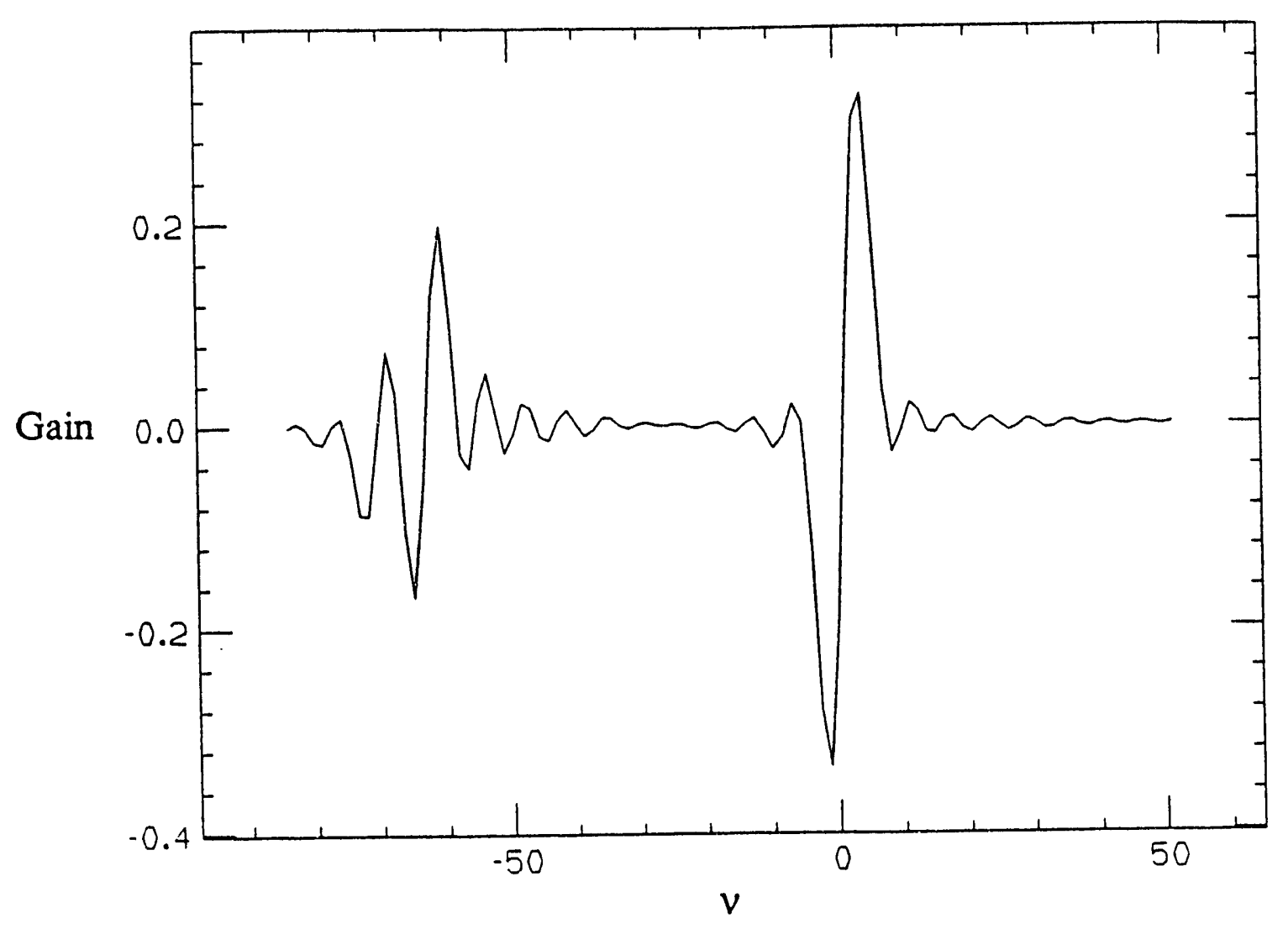

Fig.12, FEL Gain vs $v$ from Madey 's Theorem $\left[v=2 \pi N\left(\omega-\omega_{1}\right) / \omega_{1}\right]$ 

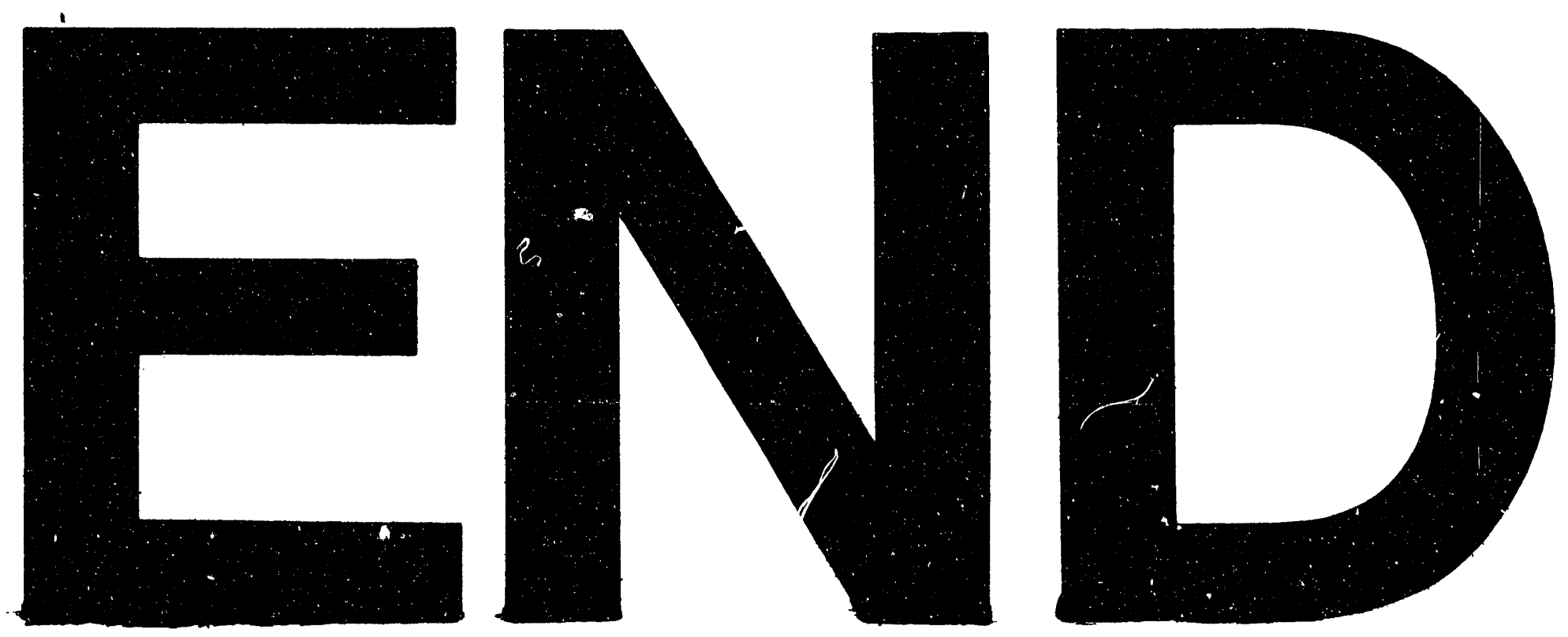

$x$

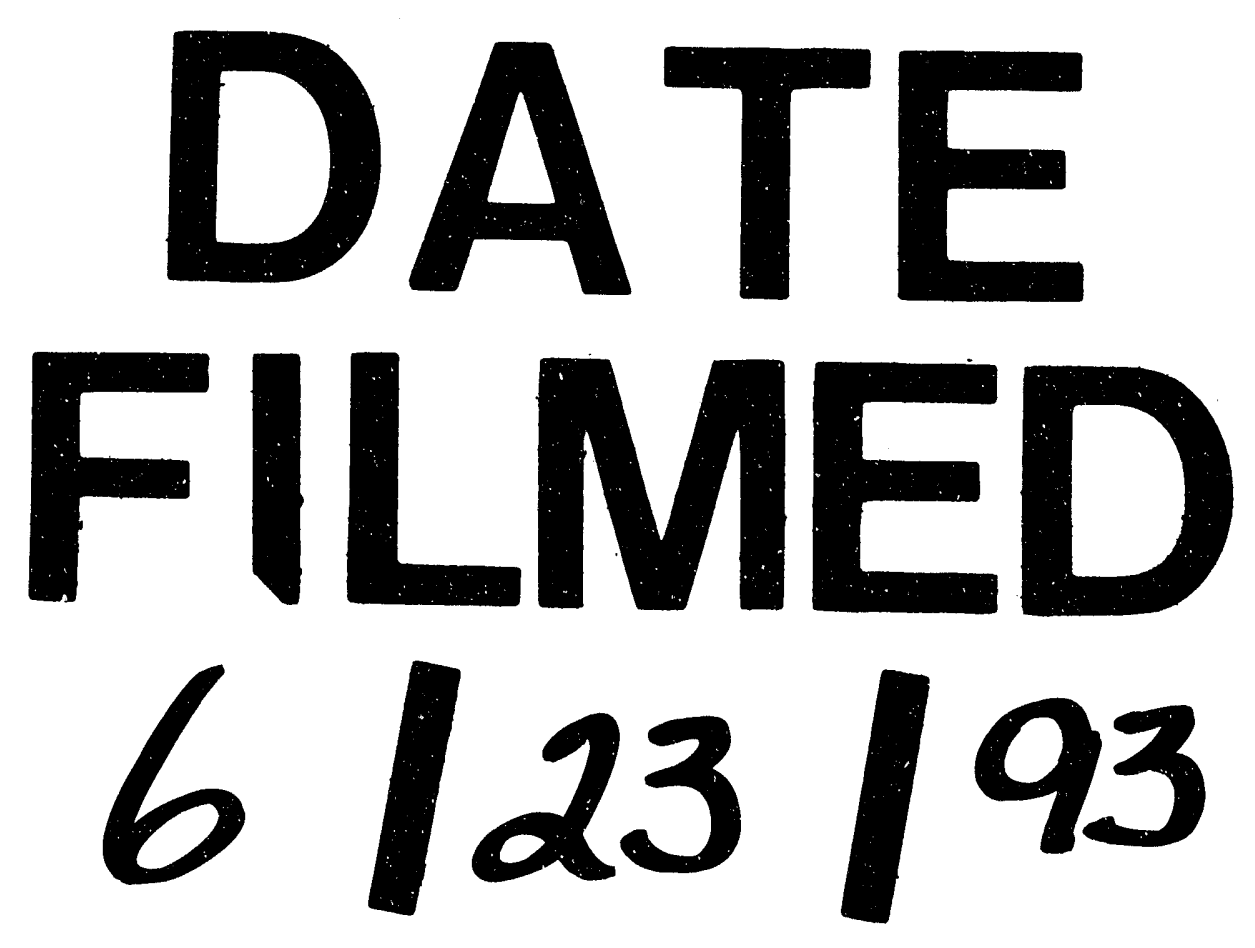


